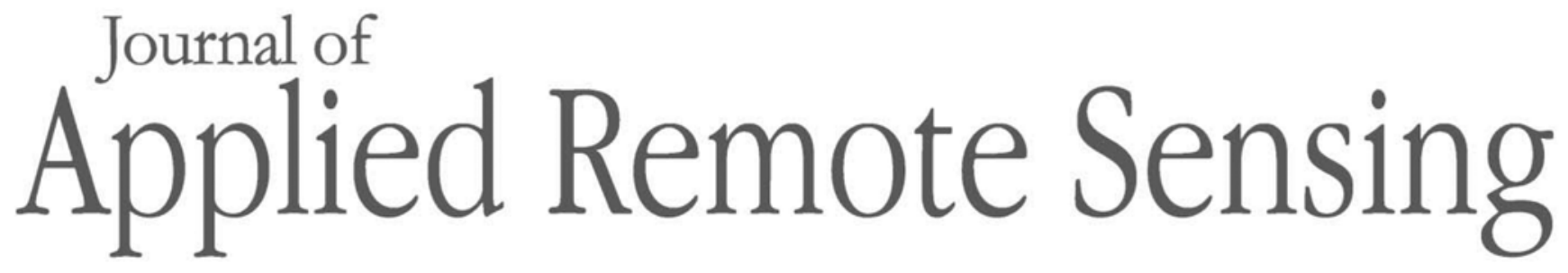

RemoteSensing.SPIEDigitalLibrary.org

\title{
Local region power spectrum-based unfocused ship detection method in synthetic aperture radar images
}

Xiangfei Wei
Xiaoqing Wang
Jinsong Chong 016026 (2018), doi: 10.1117/1.JRS.12.016026. 


\title{
Local region power spectrum-based unfocused ship detection method in synthetic aperture radar images
}

\author{
Xiangfei Wei, ${ }^{a, b, c}$ Xiaoqing Wang, ${ }^{\mathrm{d}, *}$ and Jinsong Chong, ${ }^{\mathrm{a}, \mathrm{b}, *}$ \\ ${ }^{a}$ National Key Laboratory of Science and Technology on Microwave Imaging, Beijing, China \\ ${ }^{\mathrm{b}}$ Chinese Academy of Sciences, Institute of Electronics, Beijing, China \\ ${ }^{c}$ University of Chinese Academy of Sciences, School of Electronics, Electrical and \\ Communication Engineering, Beijing, China \\ ${ }^{\mathrm{d}}$ Institute of Microelectronics of Chinese Academy of Sciences, Beijing, China
}

\begin{abstract}
Ships on synthetic aperture radar (SAR) images will be severely defocused and their energy will disperse into numerous resolution cells under long SAR integration time. Therefore, the image intensity of ships is weak and sometimes even overwhelmed by sea clutter on SAR image. Consequently, it is hard to detect the ships from SAR intensity images. A ship detection method based on local region power spectrum of SAR complex image is proposed. Although the energies of the ships are dispersed on SAR intensity images, their spectral energies are rather concentrated or will cause the power spectra of local areas of SAR images to deviate from that of sea surface background. Therefore, the key idea of the proposed method is to detect ships via the power spectra distortion of local areas of SAR images. The local region power spectrum of a moving target on SAR image is analyzed and the way to obtain the detection threshold through the probability density function (pdf) of the power spectrum is illustrated. Numerical $P$ - and $L$-band airborne SAR ocean data are utilized and the detection results are also illustrated. Results show that the proposed method can well detect the unfocused ships, with a detection rate of $93.6 \%$ and a false-alarm rate of $8.6 \%$. Moreover, by comparing with some other algorithms, it indicates that the proposed method performs better under long SAR integration time. Finally, the applicability of the proposed method and the way of parameters selection are also discussed. (C) The Authors. Published by SPIE under a Creative Commons Attribution 3.0 Unported License. Distribution or reproduction of this work in whole or in part requires full attribution of the original publication, including its DOI. [DOI: 10.1117/1.JRS.12.016026]
\end{abstract}

Keywords: local region; power spectrum; synthetic aperture radar complex images; ship detection.

Paper 170896 received Oct. 16, 2017; accepted for publication Jan. 30, 2018; published online Feb. 20, 2018.

\section{Introduction}

Ship detection in synthetic aperture radar (SAR) images is of great significance in fishery management, maritime traffic control, marine resource exploitation, and marine environmental monitoring. ${ }^{1-4}$ Ships are usually metal-made and appear as bright targets on SAR intensity images, while sea clutter is generally presented as dark background. Consequently, ship detection is in general a problem of detecting bright targets from dark background. ${ }^{5}$ According to this principle, algorithms aimed at detecting bright points on a dark background have been well studied in Refs. 6-11. The most widely used is the constant false-alarm rate (CFAR) algorithm, ${ }^{8,12}$ which requires that ships should be bright targets on SAR images. However, under long SAR integration time, ships may have crossed numerous resolution cells during the synthetic aperture time and suffered from severe defocus. ${ }^{13}$ As a result, energy of the ships will be dispersed into these resolution cells, causing the ships to be weak targets on the SAR image. ${ }^{14}$ Under this circumstance, it would be very difficult for the CFAR algorithm to detect the ships.

In recent years, several approaches have been proposed for weak targets detection on SAR images. In 2008, Gambardella et al. ${ }^{15}$ put forward a simple but effective method that can be used

*Address all correspondence to: Xiaoqing Wang, E-mail: huadaqq@126.com; Jinsong Chong, E-mail: iecas_chong@163.com 
to detect small and weak ships. This algorithm takes advantage of the fact that a ship can be regarded as a dominant scatterer in SAR images. However, because of the long integration time, the backscattered energy of the ship will be dispersed into a large number of resolution cells and the ship can no longer be treated as a dominant scatterer. In 2010, Ouchi et al. ${ }^{16}$ proposed a ship detection algorithm based on the cross-correlation between different sublook images. In 2015, Marino et al. ${ }^{17}$ tested the performance of a series of spectra-based ship detection algorithms, including the generalized likelihood ratio test method, ${ }^{18-20}$ sublook entropy method, ${ }^{21}$ sublook coherence method, ${ }^{1,22}$ and sublook cross-correlation method, ${ }^{23,24}$ through different space-borne SAR data. All of the aforementioned algorithms are based on the assumption that a ship can be considered as a coherent scatterer so that it possesses higher degree of coherence than the uncorrelated sea clutter in different sublook images. However, under long integration time, ships will be seriously defocused and present low coherence on SAR images. Therefore, the aforementioned algorithms may no longer be effective. ${ }^{17}$

In this paper, a ship detection algorithm based on local region power spectra of SAR complex images is proposed. In general, sea surface can be treated as having a flat spectrum in the bandwidth acquired by the SAR sensor. Therefore, the shape of the power spectrum of sea clutter is expected to be the same as the antenna's two-way power pattern. ${ }^{25}$ Under long synthetic aperture time, ships may have crossed numerous resolution cells during the integration time and its backscattered energy will disperse into these resolution cells. For a small local area that contains a small fraction of the defocused ship, Doppler history of the ship will account for a small portion of the entire SAR spectrum as the ship is only illuminated by a portion of the SAR antenna beam. As a result, the power spectrum of this local area will be distorted and deviates from the SAR two-way power pattern due to the ship. Therefore, the proposed method is able to detect the ships by detecting the distortion of the local region power spectra of SAR complex images. When conducting ship detection, SAR complex image is first divided into different image patches. Subsequently, a threshold is introduced to determine whether the power spectra of the image patches are distorted. If the power spectrum of an image patch exceeds the threshold at some frequency points, the power spectrum will be considered as distorted and a ship will be regarded as detected in that image patch.

This paper is organized as follows: the power spectrum of sea clutter and that of a moving target in different local areas of SAR image is analyzed in Sec. 2.1. Based on these analyses, the proposed method is presented in Sec. 2.2. The summary and flowchart of the proposed method are shown in Sec. 2.3. In Sec. 3.1, simulation of point targets is implemented to demonstrate the power spectra variation of moving targets in different local regions. Then, the proposed method is applied to $P$ - and $L$-band airborne SAR ocean data. Finally, the detection result and its comparison with some other methods are presented in Sec. 3.2. In Sec. 4, the applicability of the proposed method and the way of parameters selection are discussed, respectively. Finally, conclusions are made in Sec. 5.

\section{Local Region Power Spectrum-Based Ship Detection Method in SAR Images}

\subsection{Local Region Power Spectrum of Sea Clutter Moving Target}

In general, the azimuthal power spectrum of sea clutter can be regarded as having a same shape as the antenna's two-way power pattern, i.e., the azimuthal power spectrum of sea clutter and SAR two-way power pattern satisfy the following relationship: ${ }^{25}$

$$
S_{b}(f)=E\left\{\left|S_{a}(f)\right|^{2}\right\}=A \bullet G^{2}\left(f-f_{d c}\right),
$$

where $S_{b}(f)$ is the azimuthal power spectrum of sea clutter, $S_{a}(f)$ denotes the azimuthal Doppler spectrum, $E\{\bullet\}$ is the mathematical expectation, $A$ is a constant, $G(f)$ is the antenna's two-way power pattern, and $f_{d c}$ indicates the Doppler centroid. In practice, it is necessary to average the obtained azimuthal power spectrum in range direction in the presence of noises. ${ }^{25}$

For a moving target, the azimuthal signal after imaging process can be expressed as (derivation of moving target imaging can be seen from Appendix A) 


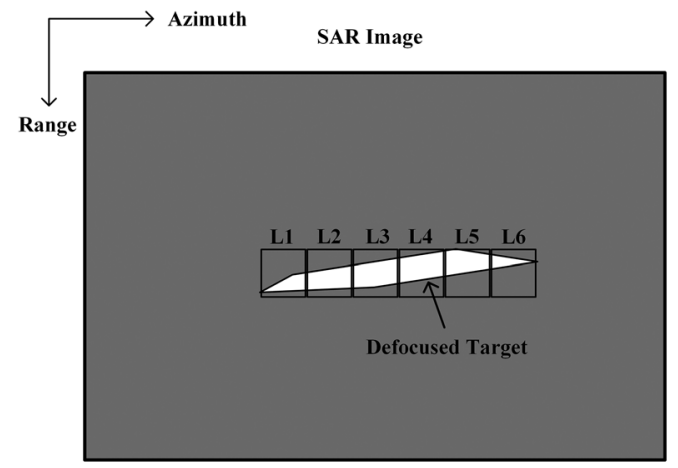

(a)

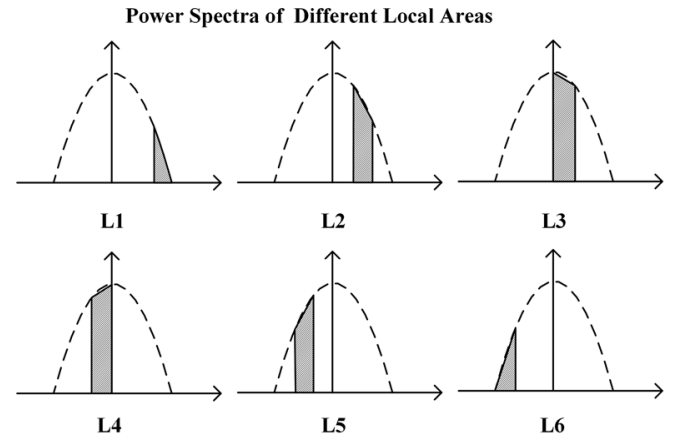

(b)

Fig. 1 Schematic of the power spectra variation of a moving target in different local areas. (a) Six local areas (L1-L6), which contain different parts of a defocused target. (b) Power spectra of the six local areas. Since the target is illuminated by different portion of the antenna beam, its power spectra occupy different parts of the total spectrum in different local areas.

$$
S(t)=G\left[\frac{\left(V_{\mathrm{SAR}}+V_{a}\right) K_{a} t}{\left(K_{a}-K_{t}\right) R}\right] \exp \left[j \pi\left(\frac{K_{t} K_{a}}{K_{a}-K_{t}}\right) t^{2}+j \pi \frac{4 V_{r}}{\lambda} t\right],
$$

where $G(\alpha)$ is the SAR two-way antenna pattern, $\lambda$ is the radar wavelength, $R$ is the slant range, $V_{\text {SAR }}$ indicates the speed of SAR platform, and $V_{a}$ and $V_{r}$ are the speeds of the moving target in azimuthal and radial directions, respectively. $K_{t}=-\frac{2\left(V_{\mathrm{SAR}}+V_{a}\right)^{2}}{\lambda R}$ indicates the frequency modulation rate of the moving target, $K_{a}=-\frac{2 V_{S A R}^{2}}{\lambda R_{0}}$ is the frequency modulation rate of a stationary target, $t \in\left[-\frac{T}{2} \frac{\left(K_{a}-K_{t}\right)}{K_{a}}, \frac{T}{2} \frac{\left(K_{a}-K_{t}\right)}{K_{a}}\right]$, and $T=\frac{\lambda R}{D\left(V_{\mathrm{SAR}}+V_{a}\right)}$ is the synthetic aperture time.

As can be seen, the moving target is defocused in azimuth. If we take a small part of the defocused target into consideration, i.e., choose a time window

$$
t^{\prime} \in\left[t_{c}-\frac{\Delta t}{2}, t_{c}+\frac{\Delta t}{2}\right]
$$

then the power spectrum of the defocused target in that time window can be expressed as

$$
S_{t}(f)= \begin{cases}G^{2}\left[-\frac{\lambda R\left(f-2 V_{r} / \lambda\right)}{2\left(V_{\mathrm{SAR}}+V_{a}\right)}\right], & f \in\left[\frac{K_{t} K_{a}}{K_{a}-K_{t}}\left(t_{c}-\frac{\Delta t}{2}\right), \frac{K_{t} K_{a}}{K_{a}-K_{t}}\left(t_{c}+\frac{\Delta t}{2}\right)\right]+\frac{2 V_{r}}{\lambda}, \\ 0, & \text { others }\end{cases}
$$

where $f$ denotes frequency. To make sure that $t^{\prime}$ is within the valid time of the moving target, $t_{c}$ and $\Delta t$ should satisfy the following inequality:

$$
-\frac{T}{2} \frac{\left(K_{a}-K_{t}\right)}{K_{a}}+\frac{\Delta t}{2}<t_{c}<\frac{T}{2} \frac{\left(K_{a}-K_{t}\right)}{K_{a}}-\frac{\Delta t}{2} .
$$

From Eqs. (4) and (5), it can be seen that Doppler history of the moving target in that time window only accounts for a small portion of the entire SAR bandwidth, which results from the fact that the moving target is illuminated by a small portion of the antenna beam in that time window. This phenomenon is shown in Fig. 1. As can be seen from Fig. 1(a), six local areas (L1-L6), which contain different parts of the defocused target, are taken from the SAR image. The corresponding power spectra are shown in Fig. 1(b). It can be seen that at different local areas, power spectra of the moving target only occupy a small fraction of the total bandwidth with a different Doppler centroid.

\subsection{Local Region Power Spectrum-Based Ship Detection Method}

When the integration time of SAR system is long, a ship may have crossed numerous resolution cells during the synthetic aperture time. Despite that a ship may have much higher scattering 
coefficient than that of sea clutter, the signal-to-clutter ratio (SCR) of the ship on an SAR intensity image is rather low since its energy is dispersed into these resolution cells. If we consider a small local area on an SAR ocean image that comprises of sea clutter and a small fraction of an unfocused ship, the Doppler history of sea clutter accounts for the entire SAR spectrum as sea clutter is completely illuminated by the antenna beam. However, as stated in Sec. 2.1, while the ship in that local area is illuminated by a small portion of the antenna beam, the Doppler history of the ship is only a fraction of the SAR spectrum. Therefore, in Doppler domain, the spectrum of sea clutter will fill the entire SAR band while the spectrum of the ship will occupy a small part of the band [c.f., Eq. (4)]. Assuming that the energy of the ship is distributed among $N^{\prime}$ different azimuthal resolution cells, while the local area contains only $n\left(n \ll N^{\prime}\right)$ of the $N^{\prime}$ resolution cells and supposing that the azimuthal bandwidth of SAR system is $B_{a}$, then the bandwidth of sea clutter is $B_{a}$ and the bandwidth of the ship approximately equals to $\frac{n B_{a}}{N^{\prime}}$. Since $n \ll N^{\prime}$, it can be seen that the spectral energy of the ship is not dispersed but concentrated in a small band. Supposing that in this local area, the energy of sea clutter is $E_{c}$ and the energy of the ship is $E_{s}$, then the SCR of the ship on SAR intensity image can be expressed as

$$
\mathrm{SCR}_{1}=\frac{E_{s}}{E_{c}} .
$$

However, if we consider the narrow band in which the ship's spectral energy is concentrated, the SCR of the ship can be approximated as

$$
\mathrm{SCR}_{2}=\frac{E_{s} /\left(n B_{a} / N^{\prime}\right)}{E_{c} / B_{a}}=\left(\frac{E_{s}}{E_{c}}\right)\left(\frac{N^{\prime}}{n}\right)=\frac{N^{\prime}}{n} \mathrm{SCR}_{1} .
$$

According to Eq. (7), the SCR of the ship will be significantly improved in Doppler domain than in intensity domain. This effect can be shown in Fig. 2. Figure 2(a) is an SAR image containing a defocused ship. In Fig. 2, frame A contains only sea clutter while frame B comprises of both sea clutter and a fraction of the defocused ship. Figure 2(b) shows the normalized power spectra of frame $\mathrm{A}$ and frame $\mathrm{B}$, where the blue curve corresponds to frame $\mathrm{A}$ and the red curve corresponds to frame B, respectively. From Fig. 2(a), it can be seen that the energy of the ship is distributed into different resolution cells, resulting in a low SCR on the intensity image. However, as can be seen from Fig. 2(b), while the energy of the ship is concentrated between the 250th and the 260th frequency points in Doppler domain, the SCR of the ship is much higher. Therefore, it is possible to detect the unfocused weak ships from Doppler domain. According to Sec. 2.1, the power spectrum of sea clutter holds a same shape as the antenna's two-way power pattern. However, due to the ships, the power spectrum may be distorted notably and will deviate from that of sea clutter at some frequency points [e.g., see Fig. 2(b)]. Consequently, a detection threshold can be set in Doppler domain. If the power spectrum of a local area exceeds the detection threshold at some frequency point, it can be considered that a ship exists in that area.

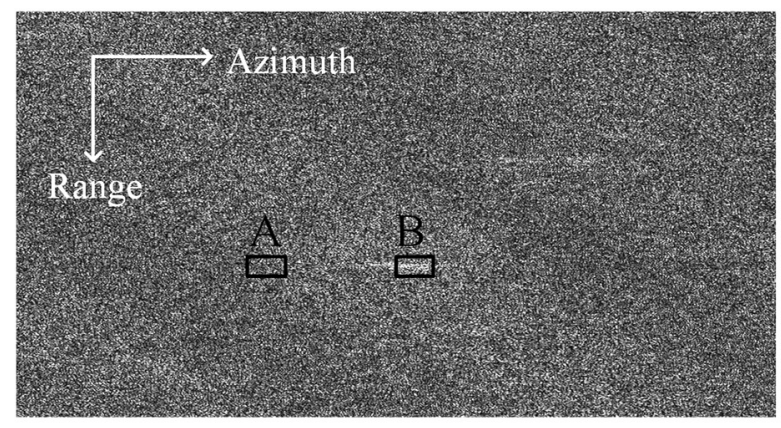

(a)

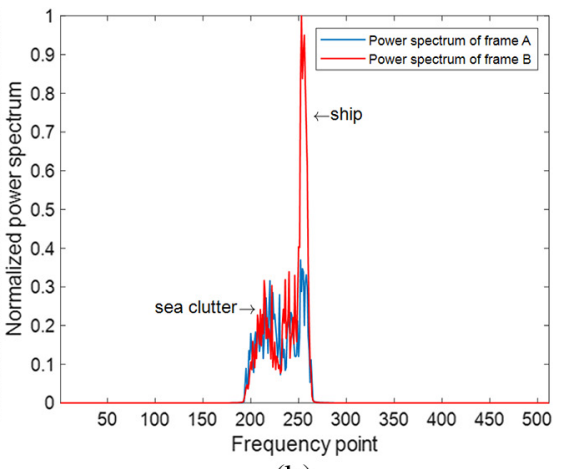

(b)

Fig. 2 Power spectrum of sea clutter and ship area. (a) Example of an SAR image containing a defocused ship and (b) normalized power spectrum of frame A (blue line, sea clutter) and frame $B$ (red line, ship area). 
Generally speaking, the probability density function (pdf) of the power spectrum of sea clutter can be described by a Gamma distribution (more details about this can be seen form Appendix B). For Gamma distribution, the probability density function can be expressed $\mathrm{as}^{26-28}$

$$
p\left(y_{i}\right)=\frac{1}{\Gamma(L)}\left[\frac{L}{S_{b}\left(f_{i}\right)}\right]^{L} y_{i}^{L-1} \exp \left[-\frac{L y_{i}}{S_{b}\left(f_{i}\right)}\right]
$$

where $y_{i}$ indicates the power frequency $f_{i}, \Gamma(\bullet)$ represents the Gamma function, $L$ is the number of averaged range bins, $S_{b}\left(f_{i}\right)$ is the mean value of $y_{i}$. Since $y_{i}$ is random, for each frequency point, a threshold $Y_{i}$ can be introduced to make sure that the probability $p\left(y_{i}>Y_{i}\right)$ does not exceed $\alpha$, i.e.,

$$
p\left(y_{i}>Y_{i}\right) \leq \alpha
$$

where $0<\alpha<1$. According to the characteristic of Gamma distribution, ${ }^{29} p\left(y_{i}>Y_{i}\right)$ can be expressed as

$$
p\left(y_{i}>Y_{i}\right)=\sum_{n=0}^{L-1} \frac{1}{n !}\left[\frac{L Y_{i}}{S_{b}\left(f_{i}\right)}\right]^{n} \exp \left[-\frac{L Y_{i}}{S_{b}\left(f_{i}\right)}\right]
$$

Therefore, if the value of $\alpha$, the power spectrum of sea clutter $S_{b}(f)$, and other related parameters are given, the detection threshold $S_{g}(f)$ can be obtained according to Eq. (10), where

$$
S_{g}\left(f_{i}\right)=Y_{i}
$$

As can be seen, $\alpha$ is an important parameter that can directly affect the detection threshold.

In ship detection, the result is inevitably affected by sea clutter, and fake targets will arise. Since sea clutter can be regarded as a stochastic process, the resulting spectrum distortion caused by sea clutter is also random. On the contrary, the motion of a ship is continuous and will cause the power spectra of several adjacent local regions to be distorted. Therefore, if a defocused ship is detected in a local region, it should also be detected in some adjacent local areas. More precisely, a detected target is reserved in the final result when the following conditions are met: (1) The power spectrum of a local area in SAR image exceeds the threshold at some frequency point and (2) in addition to this local area, power spectra distortion are also observed in no less than $Q$ adjacent local areas. As can be seen, the false-alarm rate of the proposed method is affected by $\alpha$ and $Q$. For a single local area, the false-alarm rate can be expressed as

$$
P_{f a}^{\prime}=1-\alpha^{n}
$$

where $P_{f a}^{\prime}$ is the false-alarm rate, $n$ is the azimuthal length of the local area (i.e., the length of power spectrum). However, only if a fake target occurs in more than $Q$ adjacent areas will it be reserved in final result. Therefore, the false-alarm rate is

$$
P_{f a}=\left(P_{f a}^{\prime}\right)^{Q}=\left(1-\alpha^{n}\right)^{Q} .
$$

\subsection{Flow Chart of the Proposed Method}

The proposed algorithm mainly comprises of four steps: SAR image partition, threshold calculation, ship detection, and false alarms removal, as can be seen from Fig. 3 .

The first step is to divide the SLC image into different image patches. As shown in Fig. 4, if the size of the SLC image is $M \times N$ and the size of each patch is $m \times n$, the SLC image can be divided into $\lfloor M / m\rfloor \times\lfloor N / n\rfloor$ patches. As can be seen from Eq. (7), to ensure that the ship have a higher SCR in Doppler domain, a generally smaller azimuth length of the image patch (i.e., $n$ ) should be adopted. However, a small value of $n$ would broaden the spectrum of the ship owing to the windowing effect, leading the spectral energy of the ship to disperse. In this case, the SCR of 
Wei, Wang, and Chong: Local region power spectrum-based unfocused ship detection method...

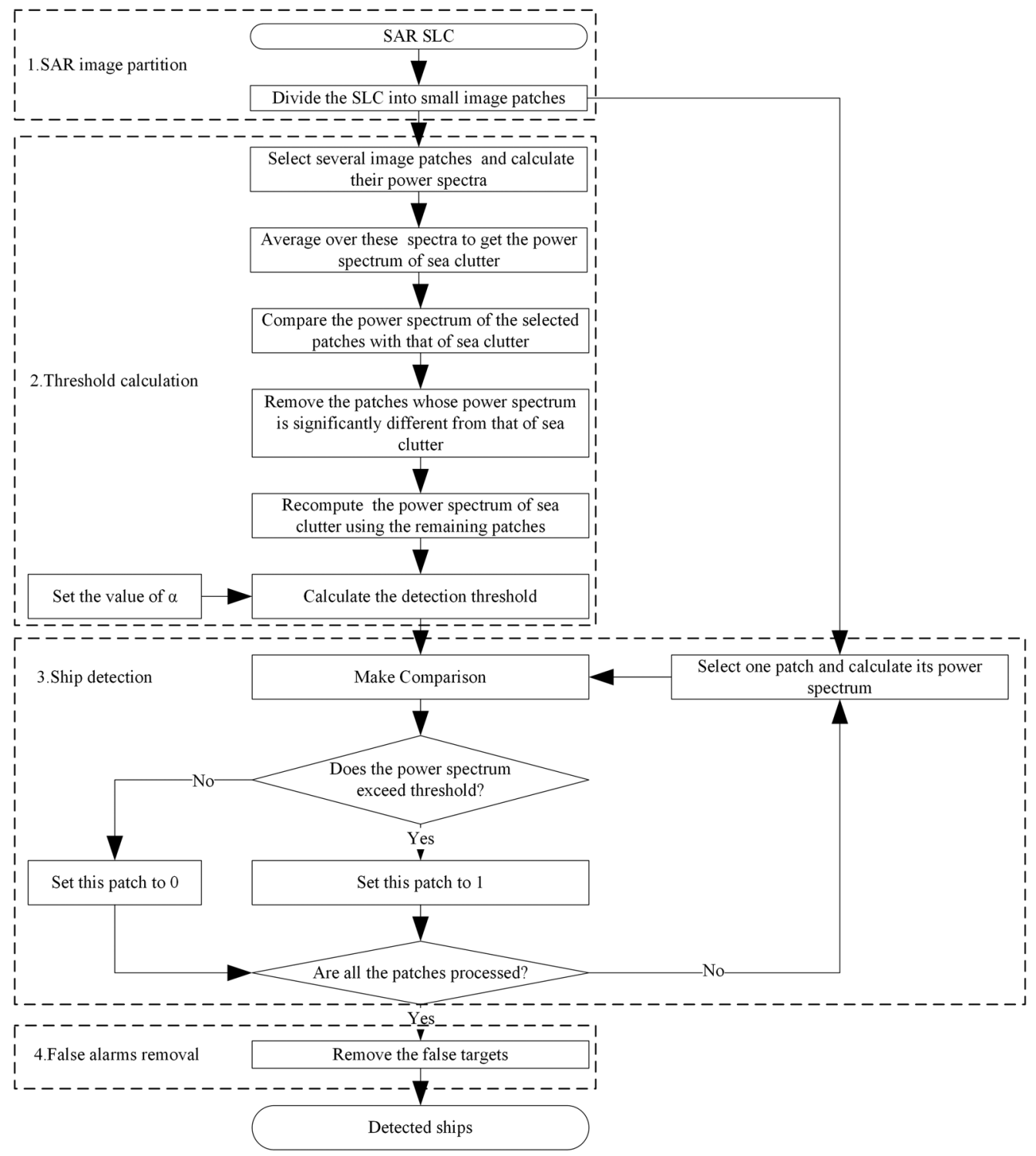

Fig. 3 Flowchart of the proposed method.

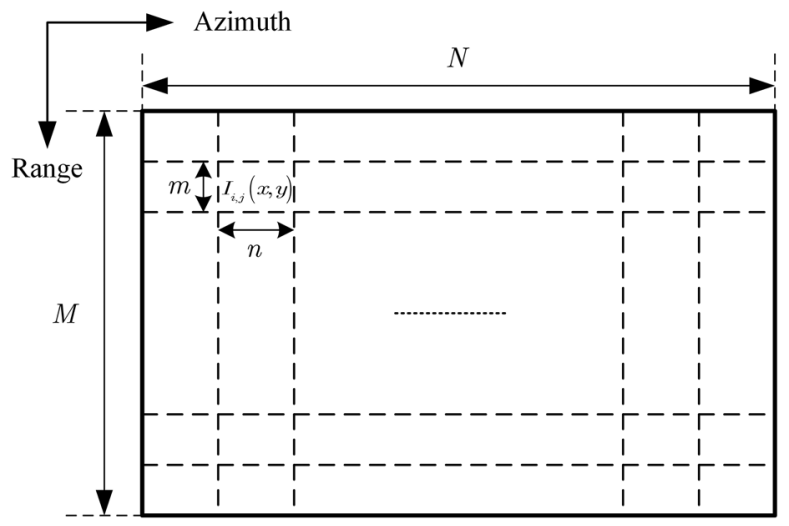

Fig. 4 Schematic diagram of SLC partition. 
the ship would be decreased. Therefore, the azimuth length of the image patch needs to be properly selected. In this paper, $n$ is generally chosen to be one-tenth of the number of resolution cells occupied by the ship in azimuth, which can be estimated based on the integration time of SAR system, the typical velocity of ships, and the azimuthal resolution of SAR image. Due to the different resolutions of SLC image in range and azimuth directions, the lengths of the image patch in range and azimuth directions are also different, i.e., $m \neq n$. Generally, $m$ is selected to make sure that the patch covers a same distance in azimuth and range directions.

The second step is to calculate the detection threshold. In this step, several image patches are randomly selected and the power spectrum corresponding to each selected patch is calculated. For an image patch, azimuthal FFT is first implemented to obtain the Doppler spectra of each range bin, which are donated by $S_{a i}(f), i=1,2, \ldots, m$. Then, the Doppler spectra are averaged to get the corresponding power spectrum

$$
S_{b}(f)=\frac{1}{m} \sum_{i=1}^{m}\left|S_{a i}(f)\right|^{2} .
$$

Subsequently, the calculated power spectra are averaged to get the spectrum of sea clutter. However, since the image patches are randomly selected, it is possible to mistakenly include the patch where a ship is located. In this case, power spectra of these randomly selected image patches are compared with that of sea clutter obtained during the last step. If the power spectra of some image patches should be significantly different from that of sea clutter, we should remove these image patches and recompute the power spectrum of the sea clutter using the remaining patches. Afterward, the detection threshold is computed through Eq. (10).

The third step is the implementation of ship detection. During this process, the power spectrum of an image patch is calculated and then compared with the threshold. If its power spectrum exceeds the threshold at some frequency points, it is considered that a ship exists in this image patch. After the same process is done for every image patch, the ships can be detected.

The final step is to remove the false alarms. As analyzed in Sec. 2.2, a ship will be reserved in the final result unless it is simultaneously detected in no less than $Q$ adjacent image patches. Through this step, some false alarms caused by sea clutter can be removed. Moreover, there are some other oceanographic and meteor phenomena that may also generate false alarms. For example, when there is a meteor phenomenon where the sea clutter is very dark in one area and very bright in the other area of the image, the proposed method may detect the bright area of sea as a target since the difference between the spectra may be very large due to the much higher power of the bright area. In this case, a preclassification is needed, i.e., the bright parts of SAR image should be identified according to their energy in intensity domain. After the SAR image is partitioned, the energy of each image patch can be estimated and a histogram of the energy values can be calculated. From this histogram, the patches with very high energy should be marked and the false alarms resulting from these marked image patches need to be excluded in the final step.

\section{Experimental Results and Analysis}

\subsection{Validation of Local Region Power Spectra Variation of Moving Targets by Simulation}

In this subsection, power spectra variation of a moving target in different SAR local regions is analyzed by simulation. The parameters of the SAR platform and moving targets are listed in Table 1. Target 1 with negative speed moves in the same direction as the platform, while target 2 with positive speed moves in the opposite direction. With the parameters in Table 1, SAR raw data is generated according to Eq. (4.39) in Ref. 30. Subsequently, the Range-Doppler Algorithm (RDA) is adopted to obtain the simulated SAR image, as shown in Fig. 5.

Figure 6(a) is an enlarged view of point target 1. As shown in Fig. 6(a), six adjacent patches are selected along the azimuthal direction. The normalized power spectra of the six patches are shown in Fig. 6(b). Figure 6(c) shows the center frequency variation between these patches. 
Wei, Wang, and Chong: Local region power spectrum-based unfocused ship detection method...

Table 1 Simulation parameters of the SAR platform and moving targets.

\begin{tabular}{lcc}
\hline \hline Parametric name & Parametric symbol & Parametric value \\
\hline Wavelength $(\mathrm{m})$ & $\lambda$ & 0.2308 \\
Platform speed $(\mathrm{m} / \mathrm{s})$ & $V_{\text {SAR }}$ & 100 \\
Platform height $(\mathrm{m})$ & $H$ & 8100 \\
Squint angle $(\mathrm{deg})$ & $\theta_{s q}$ & 0 \\
Frequency modulation rate $(\mathrm{Hz} / \mathrm{s})$ & $K_{r}$ & $2.8 \times 10^{13}$ \\
Antenna length $(\mathrm{m})$ & $D$ & 4 \\
PRF $(\mathrm{Hz})$ & $F_{a}$ & 900 \\
Near range $(\mathrm{m})$ & $R_{\text {near }}$ & 10,000 \\
Azimuthal speed of target $1(\mathrm{~m} / \mathrm{s})$ & $V_{1}$ & -10 \\
Azimuthal speed of target $2(\mathrm{~m} / \mathrm{s})$ & $V_{2}$ & 5 \\
\hline \hline
\end{tabular}

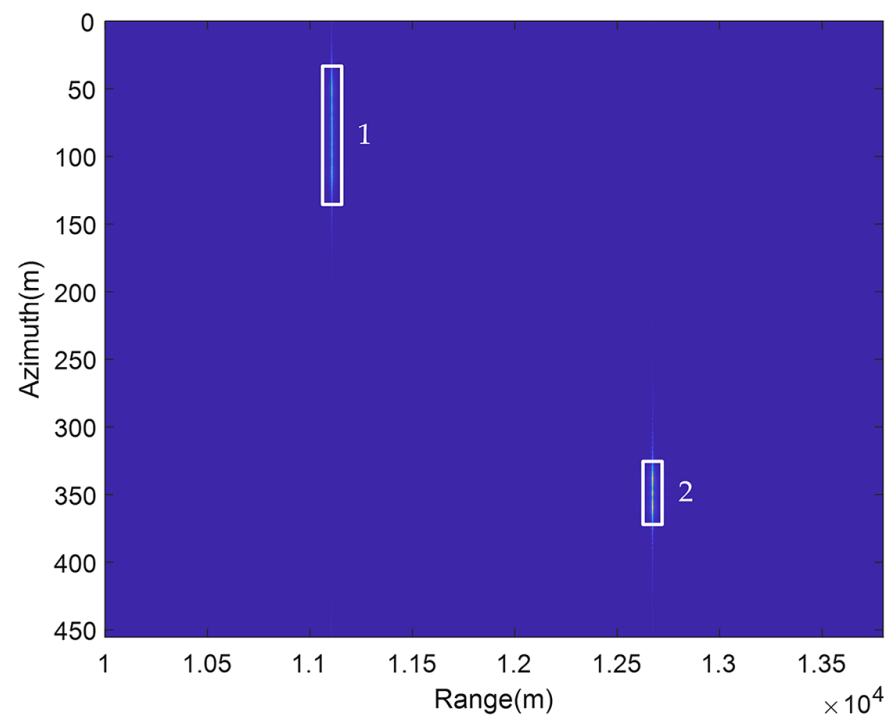

Fig. 5 SAR image of the two moving targets.

Figure $6(d)$ is an enlarged view of point target 2. Similarly, six adjacent patches are also selected along the azimuthal direction and the normalized power spectra are shown in Fig. 6(e). Figure 6(f) shows the center frequency change of target 2. From Figs. 6(a) and 6(c), it can be seen that the targets are defocused due to their azimuthal velocities. Moreover, as can be seen from Figs. 6(b) and 6(e), power spectrum of the moving targets in different local area occupies different parts of the total spectrum with different Doppler centroids, which is in consistent with the analysis in Sec. 2.1.

\subsection{Validation of the Proposed Method Using Field Data}

\subsubsection{Dataset Description}

In this section, airborne SAR ocean data were used to validate the effectiveness of the proposed method. The SAR data were obtained by the Institute of Electronics, Chinese Academy of Sciences (IECAS) in the experiment in South China Sea, 2014. In this experiment, both the 
Wei, Wang, and Chong: Local region power spectrum-based unfocused ship detection method...

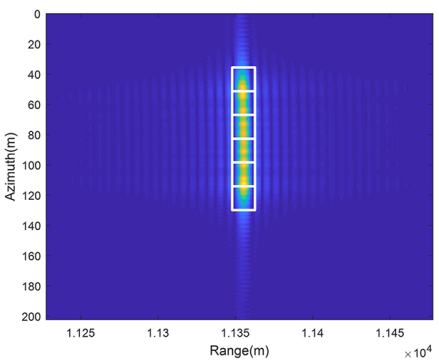

(a)

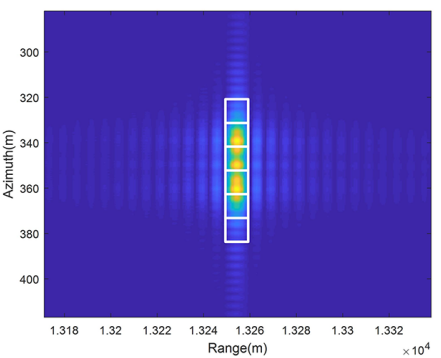

(d)

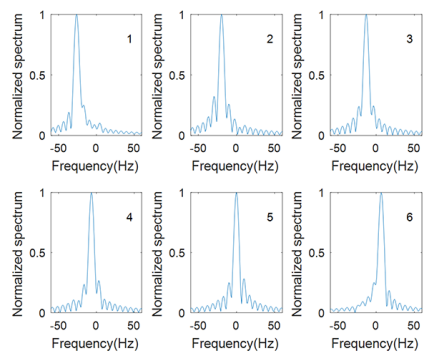

(b)

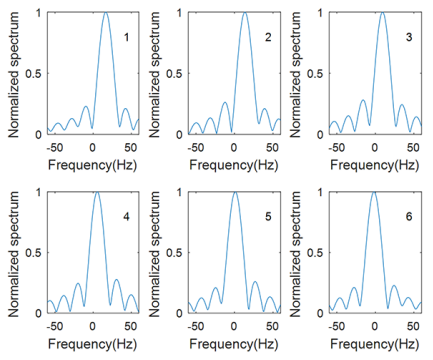

(e)

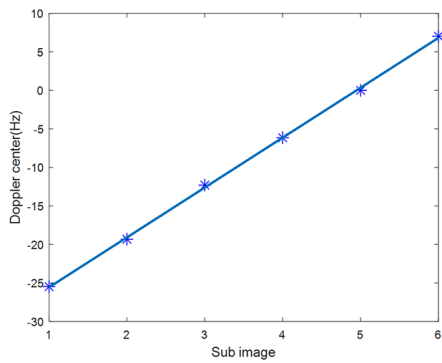

(c)

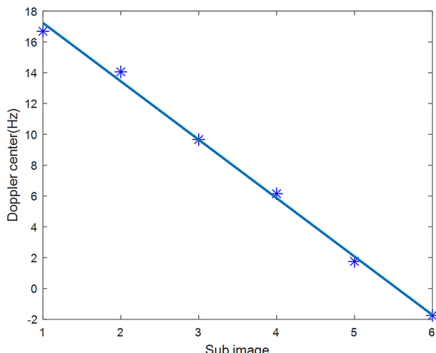

(f)

Fig. 6 Local area power spectrum analysis of moving target. (a) Enlarged view of point target 1, (b) normalized power spectrum of the six patches, (c) center frequency change between the six patches, (d) enlarged view of point target 2, (e) normalized power spectrum of the six patches, and (f) center frequency change between the six patches.

$P$-band and $L$-band SAR data were obtained. The parameters of radar system are shown in Table 2.

For SAR systems, the integration time can be expressed as ${ }^{30}$

$$
T=0.886 \frac{\lambda R}{D V_{\mathrm{SAR}}} \text {. }
$$

As can be seen, the integration time is proportional to the wavelength and $R / V$ of SAR systems. Since $P$ - and $L$-band air-borne SAR systems have relatively long wavelengths and large $R / V$,

Table 2 Parameters of the SAR system.

\begin{tabular}{lccc}
\hline \hline & & \multicolumn{2}{c}{ Parametric value } \\
\cline { 2 - 4 } Parametric name & Parametric symbol & $P$ & $L$ \\
\hline Wavelength $(\mathrm{m})$ & $\lambda$ & 0.5 & 0.23 \\
Platform speed $(\mathrm{m} / \mathrm{s})$ & $V_{\text {SAR }}$ & 120 & 132 \\
Platform height $(\mathrm{m})$ & $H$ & 8600 & 8100 \\
Squint angle (deg) & $\theta_{s q}$ & 0 & 0 \\
Frequency modulation rate $(\mathrm{Hz} / \mathrm{s})$ & $K_{r}$ & $5.3 \times 10^{9}$ & $3.9 \times 10^{13}$ \\
Antenna length $(\mathrm{m})$ & $D$ & 2 & 4 \\
PRF $(\mathrm{Hz})$ & $F_{a}$ & 1000 & 900 \\
Pulse duration $(\mathrm{ms})$ & $T_{r}$ & 0.03 & 0.0054 \\
Band width $(\mathrm{MHz})$ & $B_{r}$ & 160 & 210 \\
Polarization mode & & $\mathrm{VV}$ & $\mathrm{VV}$ \\
\hline \hline
\end{tabular}




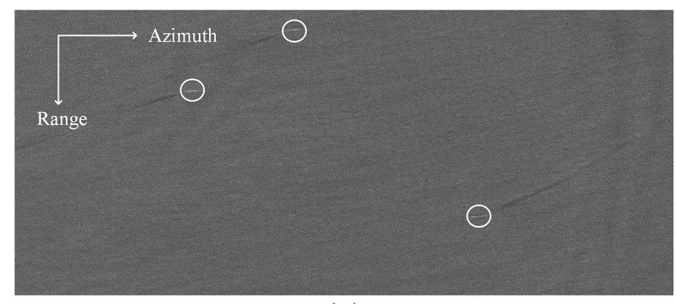

(a)

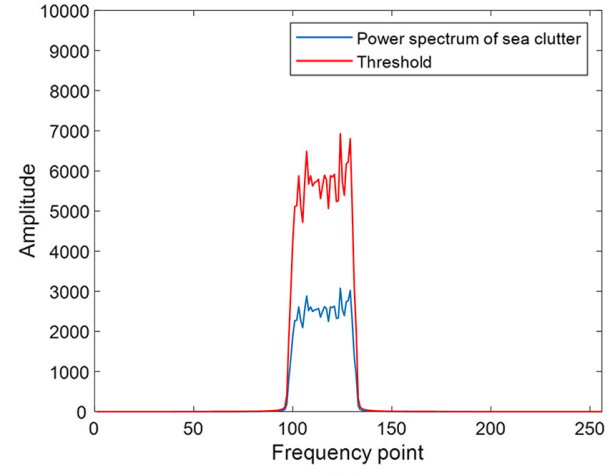

(c)

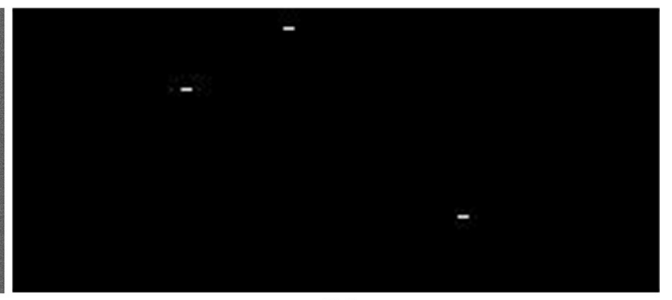

(b)

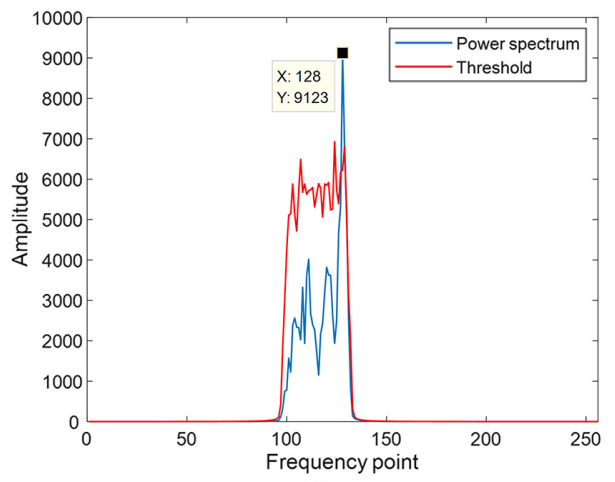

(d)

Fig. 7 Example of three weak ships and the detection result when sea condition is low. (a) Processed SAR image, (b) detection result, (c) power spectrum of sea clutter (blue line) and the corresponding threshold (red line), and (d) comparison of threshold (red line) and the power spectrum of a small patch in the center of the lower-right circle (blue line).

the SAR data used in this paper have a long integration time. Therefore, the ships are rather weak on SAR images since their energies are dispersed into numerous resolution cells.

\subsubsection{Ship Detection Using Real SAR Images}

In this subsection, the proposed method is tested on the acquired SAR images with different bands, different sea conditions, different resolutions, different target sizes, and SAR images in the presence of internal waves.

Figure 7 shows the application of the proposed method on an SAR image, where the sea condition is low. The SAR image shown in Fig. 7(a) is obtained on November 5, 2014, with a synthetic aperture time about $32 \mathrm{~s}$ ( $P$ band). The size of the image is $12,000 \times 5196$ (azimuth $\times$ range) pixels and the resolution is $0.13 \mathrm{~m} \times 0.65 \mathrm{~m}$ (azimuth $\times$ range). Three seriously defocused and poorly visible ships are found, as indicated by the white circles. The SAR image is first divided into different image patches with a patch size of $256 \times 50$ (azimuth $\times$ range) pixels. The second step is calculating the detection threshold. In this step, 20 patches are selected randomly (no ships are included in these image patches). Subsequently, power spectra of these 20 patches are averaged to reduce the influence of noises. Afterward, the corresponding detection threshold is calculated according to Eqs. (9) and (10). Here, $L=50$ and $\alpha=0.999$, and the threshold is shown in Fig. 7(c). The third step is to compare the power spectrum of each image patch with the threshold, respectively. Figure 7(d) shows the comparison of the threshold with the power spectrum of an image patch in the center of the lower right circle. It can be clearly seen that the power spectrum of this patch exceeds the threshold at the 128th frequency point. The fourth step is removing the interference caused by sea clutter. Here, $Q$ is chosen to be 4. Finally, the detection result is shown in Fig. 7(b). In this case, all of the ships are detected by the proposed method. Since each image patch results in one pixel in Fig. 7(b), the resolution of the resulting image is coarser than that of the original image. Therefore, the resulting image is up-sampled so that it keeps the same resolution as the original image. 


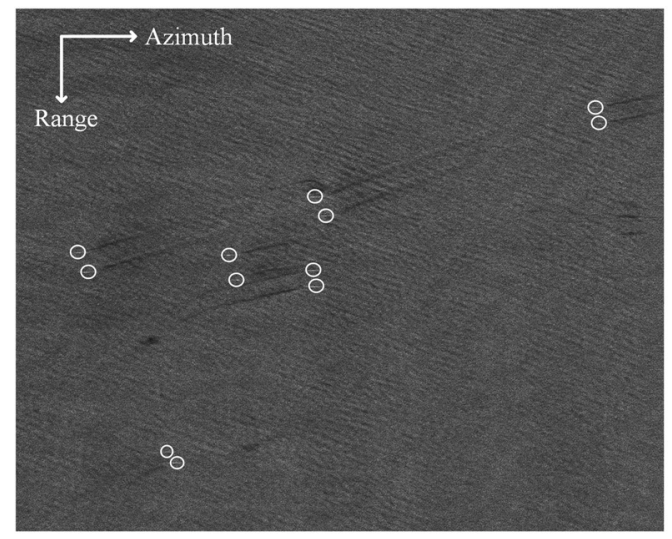

(a)

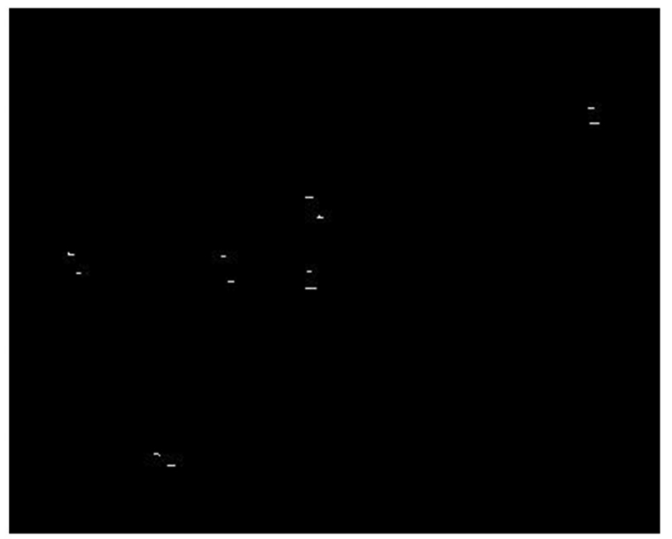

(b)

Fig. 8 Ship detection result when sea condition is high. (a) Processed SAR image and (b) detection result.

As noted in Sec. 2.2, the performance of the proposed method may be affected by sea clutter. Figure 8 shows an example when sea condition is higher. The SAR image shown in Fig. 8(a) is obtained on October 14, 2014, with a synthetic aperture time about $23 \mathrm{~s}$ ( $L$ band). The size of the image is $12,400 \times 10,000$ (azimuth $\times$ range) pixels and the resolution is $0.16 \mathrm{~m} \times 0.56 \mathrm{~m}$ (azimuth $\times$ range). Twelve ships (circled) can be identified from Fig. 8(a). The parameters for ship detection in this example are the same as those in the former example except that the size of the image patch is $128 \times 32$ (azimuth $\times$ range) pixels. The detection result is shown in Fig. 8(b). In this example, a rather good detection result is achieved by the proposed method, since all of the 12 ships are detected with no false alarms.

Oceanic internal waves are common phenomena that can be seen on SAR images, which are typical features that cause false alarms in ship detection. ${ }^{15}$ An example of the ship detection results in the presence of oceanic internal waves is shown in Fig. 9. The SAR image in Fig. 9(a) is obtained on September 13, 2014, with a synthetic aperture time about $20 \mathrm{~s}$ ( $L$ band). The size of the image is $8000 \times 5600$ (azimuth $\times$ range) pixels and the resolution is $0.16 \mathrm{~m} \times 0.56 \mathrm{~m}$ (azimuth $\times$ range). Twelve ships (circled) can be recognized from Fig. 9(a). The parameters for ship detection in this example are the same as those in the last example. Figure 9(b) is the detection result in which all of the 12 ships are detected with no false alarms either. As can be seen, the detection result is fairly good even in the presence of oceanic internal waves.

Target size is another problem that should be considered when performing the proposed method. The detection result of the proposed method on small ship targets is shown in

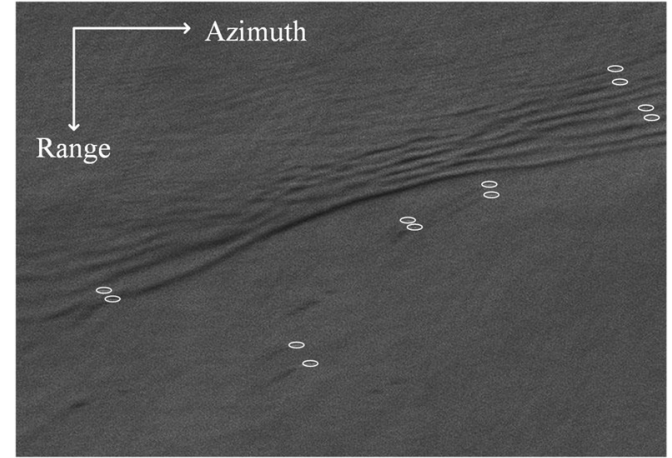

(a)

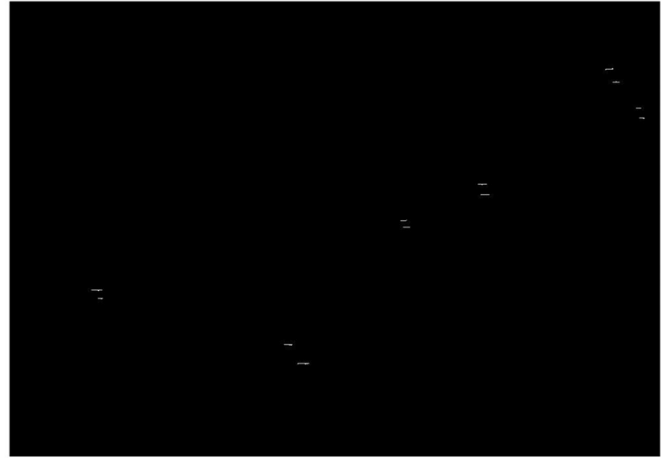

(b)

Fig. 9 Ship detection result in the presence of oceanic internal waves. (a) Processed SAR image and (b) detection result. 


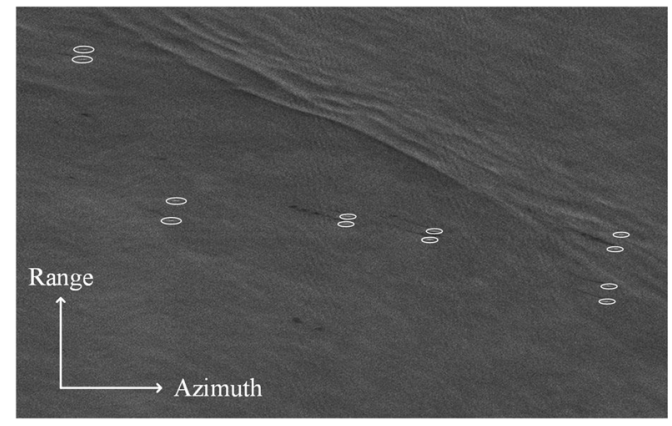

(a)

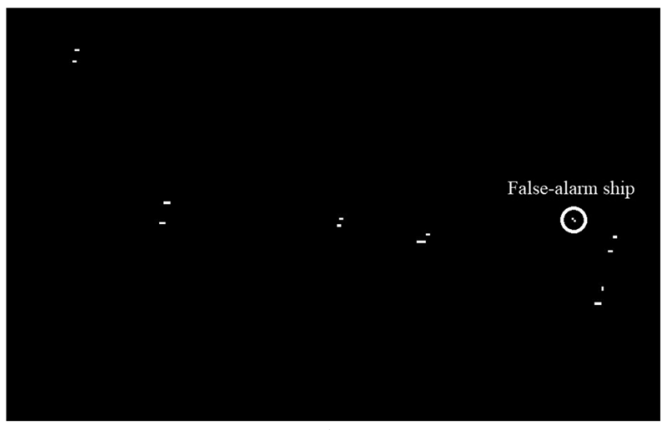

(b)

Fig. 10 Ship detection result for small targets. (a) Processed SAR image and (b) detection result.

Fig. 10. The SAR image in Fig. 10(a) is obtained on September 13, 2014, and the synthetic aperture time is about $22 \mathrm{~s}$ ( $L$ band). The size of the image is $9200 \times 5800$ (azimuth $\times$ range) pixels with a resolution of $0.3 \mathrm{~m} \times 1.2 \mathrm{~m}$ (azimuth $\times$ range). Due to the relatively coarse resolution, the identified 12 ships (circled) occupy fewer resolution cells on the image. To detect these small ships, a small patch size and a small value of $Q$ are adopted. In this example, the patch size is $64 \times 16$ (azimuth $\times$ range) pixels and $Q$ is 2 . Figure $10(\mathrm{~b})$ is the detection result of the method. As can be seen, all the ships are detected, however, with one false alarm target. The false alarm target might result from the bright return from the oceanic internal waves and was not removed due to the small value of $Q$.

\subsubsection{Comparison with Other Methods}

To further validate the effectiveness of the proposed method, the methods proposed to tackle with ship detection in low contrast condition in Refs. 1 and 15 and the traditional CFAR algorithm are also tested on the acquired SAR data. With the intention of detecting weak targets, the threshold $T_{0}$ in CFAR algorithm is set to be 1.

To demonstrate the correctness of the detection results, ships with wake signatures are chosen to test the algorithms. For the SAR data, a total number of 93 obvious wakes are identified. The detection result of the proposed method and its comparison with the three other algorithms are summarized in Table 3. As can be seen, since the ships in this experiment are rather weak, only three ships are detected by CFAR algorithm. At the same time, due to the long integration time of SAR system, ships are seriously defocused and present poor coherence between different sublook images. Therefore, the method proposed in Ref. 1 does not perform well either. Moreover, with the rational analysis of the power spectra of the defocused ships and sea clutter in local SAR image, the method proposed in this paper can improve the detection probability from $75.3 \%$ to $93.6 \%$ and reduce the false alarm rate from $10.8 \%$ to $8.6 \%$ compared with the algorithm in Ref. 15.

Table 3 Comparison of different methods.

\begin{tabular}{lccccc}
\hline \hline & \multicolumn{2}{c}{ Detected ships } & & \multicolumn{2}{c}{ False alarm ships } \\
\cline { 2 - 3 } \cline { 5 - 6 } Methods & Number & Detection rate (\%) & & Number & False-alarm rate (\%) \\
\hline CFAR algorithm & 3 & 3.2 & 12 & 12.9 \\
Method in Ref. 1 & 60 & 64.5 & 15 & 16.1 \\
Method in Ref. 15 & 70 & 75.3 & & 10 & 10.8 \\
Proposed method & 87 & 93.6 & 8 & 8.6 \\
\hline \hline
\end{tabular}




\section{Discussions}

\subsection{Analysis of the Applicability of the Method}

The proposed algorithm is mainly aimed at the detection of seriously defocused and consequently poorly visible ships on SAR images. Assume that the length of the ship is $L_{s}$ and its azimuthal speed and radial speed are $V_{a}$ and $V_{r}$, the azimuth and range length of the image patch are $N_{a}$ and $N_{r}$ and the integration time of SAR system is $T$. According to Sec. 2.2, ships can be correctly detected at least when one of the following conditions is satisfied:

$$
V_{a} \bullet T+L_{s} \geq Q \bullet N_{a} \quad V_{r} \bullet T+L_{s} \geq Q \bullet N_{r} .
$$

When the velocity of ships is slow and the synthetic aperture time is short, ships are well focused. Therefore, the power spectra of local regions where a ship is located will be much larger than that of sea clutter. If the ships are large enough so that Eq. (16) can be satisfied, they can be accurately detected by the proposed method. In other words, the proposed method can be also applied to the well-focused ships. However, there is a specific case that should be noted. This method may fail when the ships are both small and well-focused. Since a small and well-focused ship covers only a few resolution cells on SAR image so that Eq. (16) is not satisfied. As stated in Sec. 2.2, this ship will be removed as interference caused by sea clutter. Nonetheless, when well focused, ships present as bright targets on SAR images. In this circumstance, traditional detection algorithms based on SAR intensity image, such as CFAR, can be used.

The proposed algorithm is tested on $P$ - and $L$-band air-borne SAR ocean images and results show that the proposed method can well detect the weak ships. As can be seen from Eq. (16), this method can be used when the energy of ships is distributed numerous resolution cells, which requires long integration time of SAR system or fast speeds of ships. However, for typical Low Earth Orbit SAR (LEO SAR), e.g., Radarsat-2 and Sentinel-1, the integration time is only several seconds owing to the small $R / V$. Moreover, most ships move at a relatively slow speed. Consequently, ships occupy few resolution cells on LEO SAR images. Therefore, the proposed method has limited application on LEO SAR images. However, for Medium Earth Orbit SAR (MEO SAR) or Geostationary SAR (GEO SAR), the integration time is much longer. ${ }^{31}$ In this situation, even if their speeds are slow, ships will cross numerous resolution cells and cause the power spectra of several adjacent local areas to be distorted. Therefore, the proposed method can be applied to the MEO SAR or GEO SAR ship detection.

\subsection{Analysis of the Parameters Selection}

From Eqs. (9) and (10), it can be seen that the detection threshold $S_{g}(f)$ is influenced by $\alpha$. In ship detection, the false targets are mainly caused by sea clutter, especially when sea state is high. Generally speaking, by increasing the value of $\alpha$, the influence of sea clutter can be reduced and the false-alarm rate can be decreased. Consequently, to reduce the false-alarm rate, it is possible to increase the value of $\alpha$ when the sea state is high. Nonetheless, when the sea state is low, the value of $\alpha$ can be appropriately reduced to improve the detection rate. The parameter $Q$ is another factor that affects the performance of the proposed method. As can be seen from Eq. (13), a higher value of $Q$ will reduce the false-alarm rate. However, a higher value of $Q$ is not appropriate detecting ships that are both small and slow-moving, since small and slow-moving ships cover few resolution cells on SAR image. As stated in Sec. 2.2, if the energy of a ship is dispersed into less than $Q$ adjacent local areas, it would be removed from the detection result as an interference. In this paper, $Q$ is chose to be 2 to 4 to make sure that the false-alarm rate is low and some small ships can also be detected.

Figure 11 shows the false-alarm rate at different values of $\alpha$ with different azimuthal lengths ( $n$ ) of the patches and different values of $Q$. As can be seen, in addition to $\alpha$ and $Q$, the azimuthal length of the patch is another important parameter that affect the false-alarm rate. A smaller value of $n$ will reduce the false-alarm rate and is more appropriate for detecting the small and slowmoving ships. However, a small value of $n$ will broaden the spectrum of the ship in Doppler domain, which is considered as inappropriate for ship detection. As stated in Sec. 2.3, $n$ is 
Wei, Wang, and Chong: Local region power spectrum-based unfocused ship detection method...

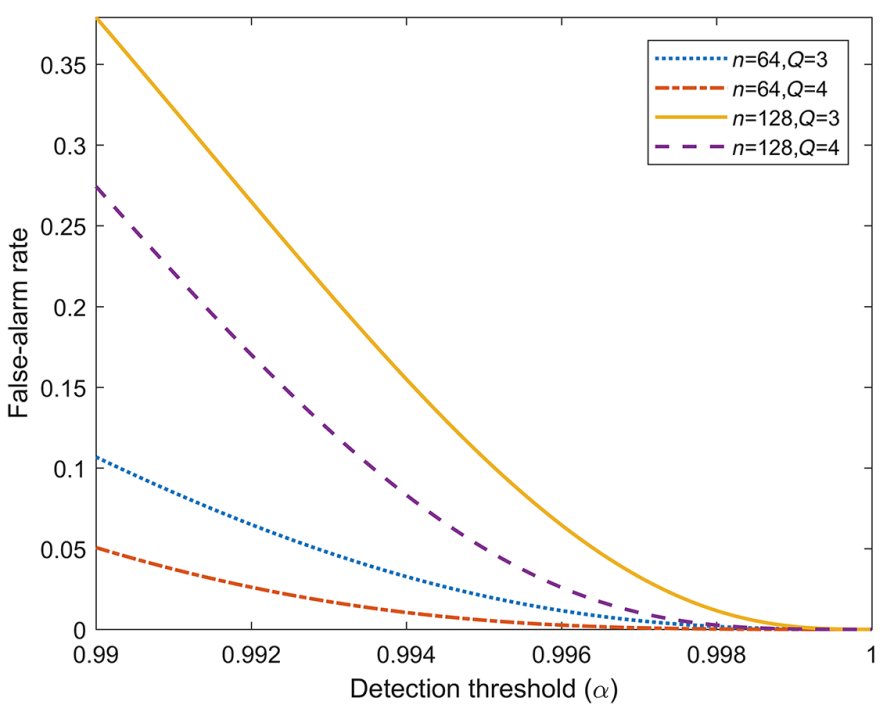

Fig. 11 False-alarm rate at different threshold value $\alpha$ with different $n$ and $Q$.

decided according to the integration time of SAR system, the typical velocity of ships, and the resolution of the image.

\section{Conclusions}

Under long SAR integration time, ships will cross numerous resolution cells, suffer from severe defocusing, and may be even buried into sea clutter. In this case, the commonly used ship detection algorithms, such as CFAR algorithm, are no longer applicable. In this paper, a ship detection algorithm in SAR images based on local region power spectra is proposed. Even though ships are rather weak on SAR intensity images, they can provide higher SCR in frequency domain since their spectral energies are concentrated. The detection threshold is first obtained according to the statistical property of sea clutter. Subsequently, SAR image is divided into different image patches. The power spectrum of each patch is calculated and compared with the threshold, respectively. If the power spectrum of an image patch exceeds the threshold at some frequency points, it is regarded that a ship exists in that patch. Moreover, some false-alarms caused by sea clutter can be removed if spectral distortions are observed in less than $Q$ adjacent image patches. The proposed method is applied to $P$ - and $L$-band airborne SAR ocean data, results show that rather good performance is achieved by the proposed method. Comparison with the traditional CFAR algorithm, the methods proposed in Refs. 1 and 15 shows that the proposed method has better performance when the integration time of SAR system is long.

This algorithm is mainly aimed at the detection of seriously defocused ships. Nonetheless, the method is also applicable for well-focused ship. However, for ships that are both small and well-focused, this method may not work. In this situation, traditional SAR intensity image-based algorithms can be used. Moreover, this method has limited application on typical LEO SAR images due to the relative short integration time. However, since MEO SAR and GEO SAR have extremely long integration time, ships are seriously smeared on these SAR images. Based on the experimental results in this paper, it can be predicted that the proposed method is applicable for MEO SAR and GEO SAR ship detection.

\section{Appendix A: Derivation of SAR Moving Target Imaging}

The azimuthal signal of a moving target can be expressed as ${ }^{30}$

$$
S(t)=G\left[\frac{V_{\mathrm{SAR}}+V_{a}}{R} t\right] \exp \left[j \pi K_{t} t^{2}+j \frac{4 \pi V_{r}}{\lambda} t\right], \quad t \in\left[-\frac{T}{2}, \frac{T}{2}\right],
$$

where $\lambda$ is the radar wavelength, $R$ indicates the slant range, $V_{\mathrm{SAR}}$ denotes the speed of SAR platform, $V_{a}$ and $V_{r}$ are the speeds of the moving target in azimuthal and radial directions, 
respectively. $K_{t}=-\frac{2\left(V_{\mathrm{SAA}}+V_{a}\right)^{2}}{\lambda R}$ indicates the frequency modulation rate of the moving target. $G(\alpha)$ is the SAR two-way antenna pattern, and $\alpha$ denotes the look angle in azimuthal direction. $T=\frac{\lambda R}{D\left(V_{\mathrm{SAR}}+V_{a}\right)}$ is the synthetic aperture time, where $D$ indicates the length of SAR antenna. The Fourier transform of Eq. (17) can be written as

$$
S(f)=G\left[\frac{\left(V_{\mathrm{SAR}}+V_{a}\right)\left(f-2 V_{r} / \lambda\right)}{K_{t} R}\right] \exp \left[-j \frac{\pi}{K_{t}}\left(f-\frac{2 V_{r}}{\lambda}\right)^{2}\right], \quad f \in\left[-\frac{K_{t} T}{2}, \frac{K_{t} T}{2}\right] .
$$

The unweighted azimuthal matching filter is given as ${ }^{30}$

$$
H(f)=\exp \left(j \frac{\pi}{K_{a}} f^{2}\right)
$$

where $K_{a}=-\frac{2 V_{\text {SAR }}^{2}}{\lambda R}$ is the frequency modulation rate of a stationary target. Multiplying Eqs. (18) and (19), we can get the filtered signal as

$$
S^{\prime}(f)=G\left[\frac{\left(V_{\mathrm{SAR}}+V_{a}\right)\left(f-2 V_{r} / \lambda\right)}{K_{t} R}\right] \exp \left[-j \pi\left(\frac{1}{K_{t}}-\frac{1}{K_{a}}\right)\left(f-\frac{2 V_{r}}{\lambda}\right)^{2}\right],
$$

where $f \in\left[-\frac{K_{t} T}{2}, \frac{K_{t} T}{2}\right]$. Then, the inverse Fourier transform is applied on Eq. (20) to get the azimuthal signal of the moving target in time domain as

$$
S^{\prime}(t)=G\left[\frac{\left(V_{\mathrm{SAR}}+V_{a}\right) K_{a} t}{\left(K_{a}-K_{t}\right) R}\right] \exp \left[j \pi\left(\frac{K_{t} K_{a}}{K_{a}-K_{t}}\right) t^{2}+j \pi \frac{4 V_{r}}{\lambda} t\right],
$$

where $t \in\left[-\frac{T}{2} \frac{\left(K_{a}-K_{t}\right)}{K_{a}}, \frac{T}{2} \frac{\left(K_{a}-K_{t}\right)}{K_{a}}\right]$.

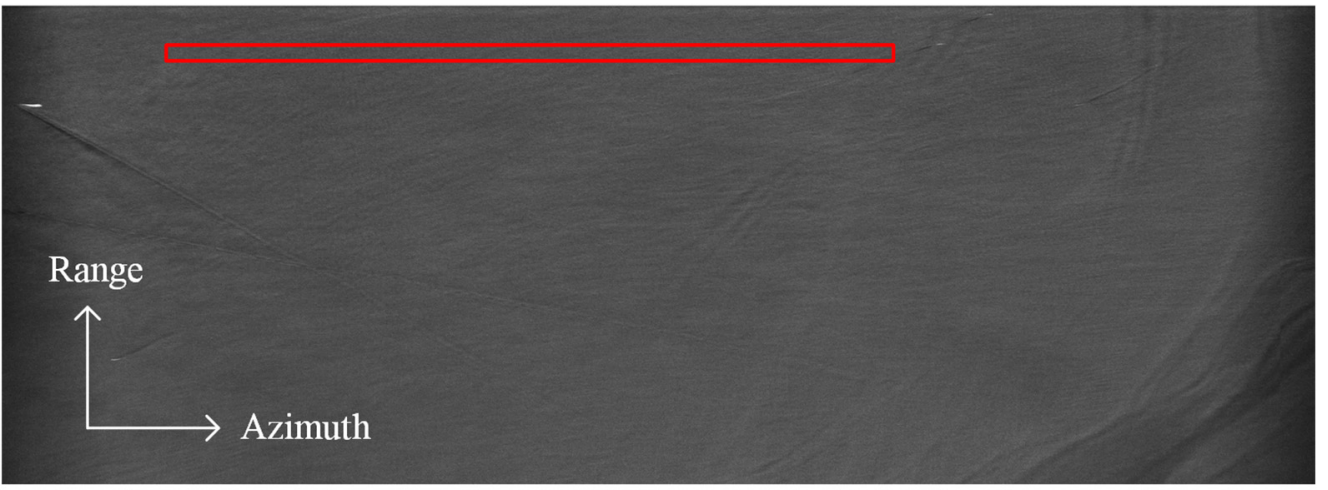

(a)

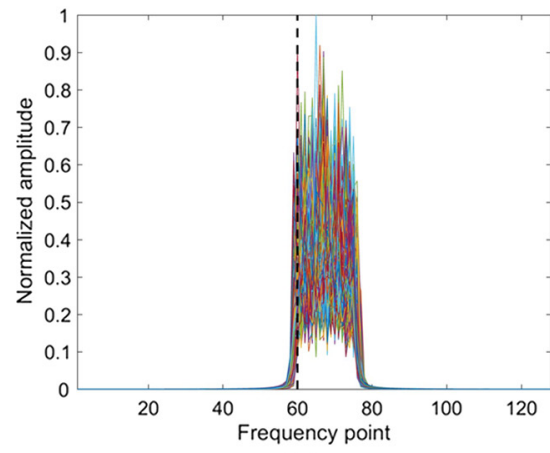

(b)

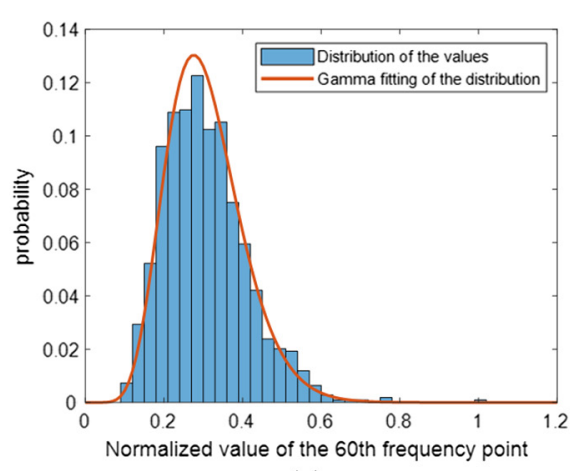

(c)

Fig. 12 Probability density function (pdf) of power spectrum of sea clutter. (a) SAR ocean image, (b) power spectra of the 1093 image patches taken from the red box, and (c) histogram of the normalized values corresponding to the 60th frequency point of the power spectra and the fitting of the distribution. 


\section{Appendix B: Analysis of the Probability Density Function of SAR Power Spectra}

For SAR systems, both the echo signal and the noise are normal distributed. Therefore, the real and imaginary parts of SAR signal are independent and identically distributed normal signals. ${ }^{32}$ In general, if the real and imaginary parts of a complex signal are independent and identically distributed normal signals, the power of the signal follows a Gamma distribution. ${ }^{26,33}$ Since Fourier transform is a linear transformation and will not change the statistical property of a random signal, a normal-distributed signal is also normal-distributed after Fourier transformation. Consequently, the real and imaginary parts of SAR spectrum are still independent and identically distributed normal signals. As a result, the probability distribution of SAR power spectrum can be described by Gamma distribution. To prove that Gamma distribution is reasonable, a histogram of the probability distribution of SAR power spectrum is shown in Fig. 12. In Fig. 12(a), about 1093 small image patches are taken from the red box along azimuthal direction. The size of the image patch is $128 \times 10$ (azimuth $\times$ range) pixels. The normalized power spectra of the image patches are shown in Fig. 12(b). Figure 12(c) is the histogram of the normalized values at the 60th frequency point of the power spectra with the Gamma fitting of the distribution (the red curve). As can be seen, Gamma distribution fits the histogram quite well.

\section{Disclosures}

The authors declare no conflict of interest.

\section{Acknowledgments}

The authors would like to thank the two anonymous reviewers for their constructive comments and recommendations.

\section{References}

1. K. Ouchi et al., "Ship detection based on coherence images derived from cross correlation of multilook SAR images," IEEE Geosci. Remote Sens. Lett. 1(3), 184-187 (2004).

2. D. J. Crisp, "The state-of-the-art in ship detection in synthetic aperture radar imagery," No. DSTO-RR-0272, Defence Science And Technology Organisation Salisbury, Info Sciences Lab, Australia (2004).

3. A. Marino et al., "Validating a notch filter for detection of targets at sea with ALOSPALSAR data: Tokyo Bay," IEEE J. Sel. Top. Appl. Earth Obs. Remote Sens. 7(12), 4907-4918 (2014).

4. R. Pelich et al., "AIS-based evaluation of target detectors and SAR sensors characteristics for maritime surveillance," IEEE J. Sel. Top. Appl. Earth Obs. Remote Sens. 8(8), 38923901 (2015).

5. J. Chong, Y. Ou, and M. Zhu, Detection of Ocean Target in Synthetic Aperture Radar Imagery, Ocean Press, Beijing (2006).

6. A. Lopes et al., "Structure detection and statistical adaptive speckle filtering in SAR images," Int. J. Remote Sens. 14(9), 1735-1758 (1993).

7. P. B. Chapple et al., "Stochastic model-based processing for detection of small targets in non-Gaussian natural imagery," IEEE Trans. Image Process. 10(4), 554-564 (2001).

8. C. C. Wackerman et al., "Automatic detection of ships in RADARSAT-1 SAR imagery," Can. J. Remote Sens. 27(4), 568-577 (2001).

9. F. DellAcqua, P. Gamba, and G. Lisini, "Rapid mapping of high resolution SAR scenes," ISPRS J. Photogramm. Remote Sens. 64(5), $482-489$ (2009).

10. S. Brusch et al., "Ship surveillance with TerraSAR-X," IEEE Trans. Geosci. Remote Sens. 49(3), 1092-1103 (2011).

11. C. Wang et al., "Ship detection for high-resolution SAR images based on feature analysis," IEEE Geosci. Remote Sens. Lett. 11(1), 119-123 (2014). 
Wei, Wang, and Chong: Local region power spectrum-based unfocused ship detection method...

12. H. Rohling, "Radar CFAR thresholding in clutter and multiple target situations," IEEE Trans. Aerosp. Electron. Syst. AES-19(4), 608-621 (1983).

13. J. Wang and X. Liu, "Velocity estimation of moving targets in SAR imaging," IEEE Trans. Aerosp. Electron. Syst. 50(2), 1543-1549 (2014).

14. A. Renga and A. Moccia, "Use of Doppler parameters for ship velocity computation in SAR images," IEEE Trans. Geosci. Remote Sens. 54(7), 3995-4011 (2016).

15. A. Gambardella, F. Nunziata, and M. Migliaccio, "A physical full-resolution SAR ship detection filter," IEEE Geosci. Remote Sens. Lett. 5(4), 760-763 (2008).

16. K. Ouchi and S.-I. Hwang, "Improvement of ship detection accuracy by SAR multi-look cross-correlation technique using adaptive CFAR," in IEEE Int. Geoscience and Remote Sensing Symp. (IGARSS '10), pp. 3716-3719, IEEE (2010).

17. A. Marino et al., "Ship detection with spectral analysis of synthetic aperture radar: a comparison of new and well-known algorithms," Remote Sens. 7(5), 5416-5439 (2015).

18. M. J. Sanjuan-Ferrer et al., "A new detection algorithm for coherent scatterers in SAR data," IEEE Trans. Geosci. Remote Sens. 53(11), 6293-6307 (2015).

19. P. Iervolino, R. Guida, and P. Whittaker, "A model for the backscattering from a canonical ship in SAR imagery," IEEE J. Sel. Top. Appl. Earth Obs. Remote Sens. 9(3), 1163-1175 (2016).

20. P. Iervolino and R. Guida, "A novel ship detector based on the generalized-likelihood ratio test for SAR imagery," IEEE J. Sel. Top. Appl. Earth Obs. Remote Sens. 10(8), 3616-3630 (2017).

21. R. Z. Schneider et al., "Polarimetric and interferometric characterization of coherent scatterers in urban areas," IEEE Trans. Geosci. Remote Sens. 44(4), 971-984 (2006).

22. A. Arnaud, "Ship detection by SAR interferometry," in IEEE Int. Geoscience and Remote Sensing Symp. (IGARSS '99), Vol. 5, pp. 2616-2618, IEEE (1999).

23. C. Brekke, S. N. Anfinsen, and Y. Larsen, "Subband extraction strategies in ship detection with the subaperture cross-correlation magnitude," IEEE Geosci. Remote Sens. Lett. 10(4), 786-790 (2013).

24. S. N. Anfinsen and C. Brekke, "Statistical models for constant false alarm rate ship detection with the sublook correlation magnitude," in IEEE Int. Geoscience and Remote Sensing Symp. (IGARSS '12), pp. 5626-5629, IEEE (2012).

25. N. Li et al., "Fast ship detection for scansar mode in wide sea areas," in IEEE Int. Geoscience and Remote Sensing Symp. (IGARSS '16), pp. 1251-1253, IEEE (2016).

26. S. Chitroub, A. Houacine, and B. Sansal, "Statistical characterisation and modelling of SAR images," Signal Process. 82(1), 69-92 (2002).

27. X. Wei et al., "Imaging algorithm of millimeter-wave LFMCW radar for water surface texture detection," J. Electron. Inf. Technol. 30(5), 1030-1035 (2016).

28. H. Meng et al., "Doppler spectrum-based NRCS estimation method for low-scattering areas in ocean SAR images," Remote Sens. 9(3), 219-240 (2017).

29. P. G. Moschopoulos, "The distribution of the sum of independent gamma random variables," Ann. Inst. Stat. Math. 37(1), 541-544 (1985).

30. I. G. Cumming and F. H. Wong, Digital Processing of Synthetic Aperture Radar Data: Algorithms and Implementation, Artech House, Norwood, Massachusetts (2005).

31. J. Li et al., "Parametric analysis and imaging method of geosynchronous SAR," Syst. Eng. Electron. 32(5), 931-936 (2010).

32. C. Oliver and S. Quegan, Understanding Synthetic Aperture Radar Images, SciTech Publishing, Raleigh, North Carolina (2004).

33. J.-S. Lee and E. Pottier, Polarimetric Radar Imaging: From Basics to Applications, CRC Press, Boca Raton, Florida (2009).

Xiangfei Wei received his BS degree from Huazhong University of Science and Technology, Wuhan, China, in 2014. Currently, he is working toward his PhD in signal and information processing in the National Key Laboratory of Science and Technology on Microwave Imaging, Institute of Electronics, Chinese Academy of Sciences, Beijing, China. He is also 
with the University of Chinese Academy of Sciences, Beijing, China. His research interest includes microwave remote sensing of the ocean.

Xiaoqing Wang received his BS degree from Xiamen University, Xiamen, China, in 2000 and his PhD from the Graduate School, Chinese Academy of Sciences, Beijing, China, in 2005. He received the President Scholarship for Outstanding Students of the Chinese Academy of Sciences in 2005. Currently, he is an associate research professor with the Institute of Microelectronics of Chinese Academy of Sciences. His research interests include microwave remote sensing of the ocean and SAR imaging.

Jinsong Chong received her BS degree from Jilin University, Changchun, China, in 1991, her MS degree from Beijing University of Aeronautics and Astronautics, Beijing, China, in 2000, and her PhD from the Graduate School, Chinese Academy of Sciences, Beijing, China, in 2003. Currently, she is a research professor with the National Key Laboratory of Science and Technology on Microwave Imaging, Institute of Electronics, Chinese Academy of Sciences. Her research interest focuses on ocean remote sensing with microwaves. 